\title{
Performance of the upgraded laser system for the Fermilab-NIU photoinjector
}

\author{
Jianliang Li*, Rodion Tikhoplav and Adrian C. Melissinos \\ Department of Physics and Astronomy, University of Rochester, Rochester, NY, 14627 \\ * Corresponding author: email, jlli@synopsys.com, 2025 NW Cornelius Pass Rd., \\ Hillsboro, OR 97124 Phone, 585-414-7028. Fax, 503-547-6017.
}




\begin{abstract}
:
The laser system for the Fermilab-NIU photoinjector [1] has been recently upgraded in order to improve reliability and to reduce amplitude fluctuations. Major modifications included the replacement of the oscillator by a diode-pumped passively mode locked Nd:YLF commercial laser [2]. The oscillator delivers 5 ps long pulses at $81.25 \mathrm{MHz}$ at an average power of $450 \mathrm{~mW}$. The number of round trips in the multi-pass amplifier was reduced by half and image relaying was introduced throughout the optical system. The frequency of the IR $(\lambda=1053 \mathrm{~nm})$ pulse was quadrupled to UV $(\lambda=263.5 \mathrm{~nm})$ by two BBO crystals. The overall efficiency for frequency quadrupling was of order $20 \%$. The shot to shot fluctuations in the UV are $\sim 5 \%$. The UV energy on the cathode is $5 \mu \mathrm{J} /$ pulse (10 $\mathrm{J} /$ pulse without the pulse stacker), yielding charge of $10 \mathrm{nC} / \mathrm{pulse}$.
\end{abstract}




\section{Introduction}

The Fermilab/NIU photoinjector [1] consists of a photoemission electron source based on an L band rf-gun. The CsTe photocathode is illuminated by an ultrashort (5 ps) UV laser pulse. The gun is followed by a 9-cell superconducting cavity, beam focusing elements to handle the $14.8 \mathrm{MeV}$ low emittance electron beam and appropriate diagnostics. Beam charge as high as $10 \mathrm{nC} /$ pulse can be delivered. A schematic overview of the photoinjector is shown in Fig. 1. In this paper, we describe the modifications made to the original laser and the performance of the upgraded laser system.

The upgraded laser system is shown in Fig. 2 and is located on two $4 \times 8$ feet optical tables. The oscillator [2] $(\lambda=1054 \mathrm{~nm})$ delivering $450 \mathrm{~mW}$ of $5 \mathrm{ps}$ long pulses at 81.25 $\mathrm{MHz}$, is phase locked to the master oscillator that controls the L-band rf. A single pulse is selected out of this train by a pulse picker [3] and amplified in a multi-pass flash lamp pumped Nd:glass laser cavity. The output of the multi-pass amplifier is further amplified in two 2-pass Nd:glass amplifiers. The frequency of the IR pulse is then doubled and quadrupled to the UV ( $\lambda=263.5 \mathrm{~nm})$, in two $10 \mathrm{~mm}$ BBO crystals. Finally the UV pulse is transported to the cathode at a distance of $20 \mathrm{~m}$ from the laser room. The laser parameters are summarized in Table I. Presently, up to 800 pulses spaced at $1 \mu$ s can be delivered at a repetition rate of $1 \mathrm{~Hz}$. Information on a similar laser system installed at the TESLA test facility at the DESY laboratory in Hamburg, Germany can be found in $[4][5]$.

An important precondition for successful operation of the photoinjector is a stable and reliable laser that delivers UV laser pulses conforming to the requirements of the linac. 
The first version of the drive laser was installed in 1998 [6] and has been in operation since then, but was limited in certain aspects. Most seriously, the chirp of the seed pulse, generated by GVD in a $2 \mathrm{~km}$ long fiber, was unstable due to environmental fluctuations. The compression ratio of the grating compressor consisting of a pair of gratings, in a fixed configuration, is dependent on the chirp of the input beam, and hence, the output pulse duration was unstable. Such pulse length instability turns into amplitude fluctuations when the frequency of the IR beam is doubled and quadrupled. Furthermore, it was difficult to maintain optimal coupling of the seed pulse into the multi-pass amplifier, requiring the use of a large number of round trips (as many as 13) to achieve the desired gain. As discussed later this contributes significantly to shot to shot fluctuations. The absence of image relay in the optical system degrades the wavefront, resulting in decreased efficiency during frequency conversion. It also made it difficult to control the profile of the UV on the cathode. In the newly upgraded system the above problems have been resolved.

In the following sections we discuss the individual components as well as the overall performance of the laser system. We begin by describing the characterization of the new seed laser. In the third section we present details on the amplification of the seed pulse in the multi-pass and 2-pass amplifiers. We also discuss the image relay optics, designed to preserve the beam waist and maintain the beam transverse profile. The IR beam waist is relayed to the doubling and quadrupling crystals so that the wavefront at the crystals is flat. The quality of the UV beam was improved by introducing a spatial filter inserted at the focal point of the UV telescope. 
The final section is divided into several subsections that cover different aspects of the system's performance. We first discuss shot to shot fluctuations. By comparing the amplitude fluctuations after the multi-pass amplifier and in the UV, we conclude that the primary source of the fluctuations is the instability of the power supply driving the flash lamp of the multi-pass amplifier. The traverse and longitudinal profiles of the UV beam, which were measured using, respectively, a CCD camera and a streak camera are presented. We also describe the UV pulse stacker and the transport line to the photocathode. Four UV pulses were stacked with a fixed time delay to construct a long pulse with a flat top in order to suppress emittance growth in the electron beam caused by space charge effects. To transport the UV beam to the photocathode, an imaging system was built, which allows us to introduce controlled transverse patterns on the electron gun. Finally, we show data from a long UV pulse train with 400 pulses. This pulse train can be extended to 800 pulses and can be made flat by preshaping the oscillator pulse train before injection into the multi-pass amplifier.

\section{Seed laser}

The new seed laser [2] provides pulses with a duration of 5 ps, and therefore there is no more need to add bandwidth and stretch the pulse.. As a result, both the long fiber and pulse compressor present in the original configuration were bypassed. , A timing box provided by the manufacturer controls the fluctuation of the seed laser phase relative to the master oscillator, and maintains the timing jitter to less than $0.2 \mathrm{ps}$.

The seed pulse was characterized in both the time and frequency domain and its output was monitored in real time to ensure that the best seed was injected to the 
amplifiers. The pulse width in the time domain was measured with a rotating-arm continuous-wave autocorrelator (CWAC) [7][8]. A 50/50 beamsplitter sends light to a fixed delay arm and to a variable delay arm, the latter consisting of a flat mirror and of a retro-reflector, mounted on a platform spinning at $13.5 \mathrm{~Hz}$. The beams from the two arms center off axis in a focusing lens, and cross each other at the focus in a thin Lithium Iodate $\left(\mathrm{LiIO}_{3}\right)$ crystal. The crossing angle of the two beams allows phase matched noncollinear second harmonic generation. The resultant green signal is detected with a photomultiplier tube (PMT) and displayed on a digital oscilloscope. A typical autocorrelation profile measured with the CWAC is shown in Fig. 3a. Assuming a Gaussian waveform for the IR pulse, the FWHM of the autocorrelation yields for the IR pulse length $5.12 \mathrm{ps}$, in close agreement with the manufacturer's specifications [2].

A commercial Optical Spectrum Analyzer (OSA) was used to monitor the spectrum of the seed laser [9]. This is a scanning grating monochromator, designed for a resolution of $0.08 \mathrm{~nm}$. The seed laser was coupled into the OSA through a $\sim 1 \mathrm{~m}$ long multi-mode fiber. The spectral waveform can be read over GPIB and recorded by computer. Figure 3b shows the spectrum of the seed laser with FWHM $0.52 \mathrm{~nm}$ as measured by the OSA.

It should be noted that the transverse profile of the seed laser was elongated in the horizontal dimension due to the fact that the Nd:YLF crystal, the gain medium of the seed laser, was cut at Brewster's angle to minimize the unwanted polarization in the output. Since the multi-pass cavity was designed for a homogeneous transverse profile, it was necessary to compensate the horizontal distortion with an anamorphic prism.

Another important issue is the timing jitter between the seed pulse and the RF signal that drives the gun and the accelerating cavity. Obviously the seed laser must be locked 
to the RF system. To achieve this goal the timing box, mentioned in the previous section, controls the phase of the seed pulse to maintain synchronization with the $81.25 \mathrm{MHz}$ reference signal; this reference is derived from the master oscillator, which controls the 1.3 GHz rf. The readout of the timing jitter is available in real time; it was less than 0.2 ps upon daily operation. $0.2 \mathrm{ps}$ jitter corresponds to a phase fluctuation of 0.094 degrees between the laser pulse and the RF signal, which in practice is completely negligible.

The seed pulse must be integrated into the entire laser system, as shown in Fig. 2. The seed laser was physically placed beside the multi-pass amplifier. After traversing the Pulse Picker (PP) and Faraday Rotator (FR), the seed laser pulse was coupled into the multi-pass cavity by a $60 \mathrm{~cm}$ focal length lens. The pulses rejected by the PP are directed to the diagnostic table, on which both the $\mathrm{CW}$ autocorrelator (CWAC) and Optical Spectrum Analyzer (OSA) are located. The output from the multi-pass amplifier is further amplified by two 2-pass amplifiers. After that, the amplified pulse is sent to the next optical table for frequency doubling and quadrupling and pulse manipulation prior to reaching the photocathode.

Because the conversion efficiency of the crystals is overwhelmingly dependant on the quality of the wavefront, it is very important to maintain the flat beam waist of the IR beam and make sure that the beam waist is properly relayed to the crystals. In addition, the UV beam after the crystals needs to be reshaped and relayed to the photocathode, which is about 20 meters away from the laser room. In the design of the system, we set the reference point of of the optical beam line at the flat mirror in the multi-pass amplifier.

\section{Amplification and conversion to UV}




\subsection{Multipass amplifier}

The explicit structure of the multi-pass cavity is shown in Fig. 4a. It is a resonator, in which the seed pulse is captured by turning on the Q-switch Pockels cell [10] and makes a certain number of passes through the amplifying medium before it is kicked out by turning off the Q-switch Pockels cell. When the amplifier works in the saturation regime, it is called a "regenerative amplifier". However, because of our requirement of amplifying up to 800 pulses to nearly the same energy, it is necessary to operate in an unsaturated mode. In this way we avoid extracting too much of the stored energy with each pulse, which would, of course, reduce the gain for the remaining pulses. We use the term "multi-pass amplifier” to indicate operation in the unsaturated regime [11].

The stable resonator cavity is formed by one flat $\left(R_{1}=\infty\right)$ and one curved $\left(R_{2}=5 \mathrm{~m}\right)$ mirror spaced $1.44 \mathrm{~m}$ apart; the optical cavity length is $157.4 \mathrm{~cm}$. For these parameters, the eigenmode of the cavity determines the size of the beam waist on the flat mirror, which is calculated to be $\sim 0.88 \mathrm{~mm}$.

In the first step of the alignment, the output from the seed laser must be coupled efficiently into the resonator cavity. Namely, the beam waist of the seed laser must be relayed onto the flat mirror in the multi-pass cavity with appropriate magnification to match the eigenmode diameter. From the product report provided by the manufacturer, the beam waist is located inside the exit window of the seed laser at a distance of $50 \mathrm{~cm}$ and the size of the beam waist there is about $0.25 \mathrm{~mm}$. A $60 \mathrm{~cm}$ focal length lens placed about $10 \mathrm{~cm}$ away from the exit window of the seed laser is adequate for good coupling of the seed pulse into the amplifier cavity. 
After propagating through the lens, PP, FR, half waveplate and being reflected by the,Brewster plate, the $5.5 \mathrm{~nJ}$ seed laser pulse (decreased to $\sim 4 \mathrm{~nJ}$ ) was injected into the multi-pass cavity (see Fig. 4a) with vertical polarization. The pulse passes through the Qswitch Pockels cell [10] that is statically biased for quarter wave, acquires a quarter-wave retardation, is reflected from the flat end mirror, and passes again through the Q-switch acquiring a further quarter wave retardation to horizontal polarization. With this polarization the pulse goes through the Brewster plate and continues through the cavity, and after one round trip through the Nd:glass rod returns to the Q-switch. At this point, in time the Q-switch has been triggered on to zero-wave retardation so that the pulse is trapped inside the cavity. The voltage on the Q-switch is held high for the length of time necessary for the desired number of round trips. After that it is triggered back to the quarter-wave retardation so that the polarization of the pulse is flipped back to vertical and the pulse is kicked out by the Brewster plate. The Faraday isolator (with permanent magnets) is used to separate the output pulse, and direct it to the 2-pass amplifiers.

The seed pulse grows rapidly in the resonator by traveling back and forth through the laser rod during the time window set by the Q-switch Pockel's cell. Figure 5 shows the single pulse amplitudes measured with a fast photodiode versus number of round trips. There are several conclusions that can be drawn from these data. First, the pulse amplitude exhibits a consistent exponential growth with an amplification factor of 3.1 for each single round trip. Second, the amplification of the pulse does not show any saturation up to 8 round trips. Third, the fact that the amplification of the pulse is evenly distributed on different round trips implies that the seed pulse was efficiently coupled into the multi-pass cavity and that the multi-pass cavity was well aligned. The overall 
energy of the single pulse after 6 round trips was measured at $6 \mu \mathrm{J}$, which represents a total gain of $\sim 1500$ in the multi-pass amplifier, or a round trip gain of 3.3. Since, as discussed below, shot to shot fluctuations are dominated by the stability of the pumping light, a small number of round trips is desired. We therefore set the time window of the Q-switch in our daily operation for 6 round trips (total time $\sim 52 \mathrm{~ns}$ ).

\subsection{Two-pass Amplifiers and UV beam}

After exiting the multi-pass amplifier, the IR pulse is coupled by the telescope system into two 2-pass amplifiers, which consist of two balanced laser rods (see Fig. 4b). The single pulse energy is multiplied by more than 25 times to $\sim 150 \mu \mathrm{J}$. The telescope is formed by two $1 \mathrm{~m}$ focal length lenses, with the flat end mirror in the multi-pass cavity at the focal plane of the first lens and the reflection mirror in the 2-pass amplifiers at the focal plane of the second lens. The other focal planes of the two lenses coincide at the midpoint between the lenses. A spatial filter may be inserted at the midpoint if it is desired to smooth out the transverse profile of the pulse emerging from the 2-pass. Similarly, another telescope formed by two $1 \mathrm{~m}$ lenses was located after the 2-pass amplifiers to relay the beam waist to the frequency conversion crystals. We achieved efficiency for frequency doubling in excess of $50 \%$ and for frequency quadrupling of up to $40 \%$. The crystal conversion efficiencies measured under different input intensity are plotted in Fig. 6. At the maximum input intensity, both crystals are in the deeply saturated region and this helps to reduce the fluctuations in the green and in the UV.

After being converted from IR (1054 nm) to UV (263 nm), the laser beam was directed to a diagnostic "target" by a telescope consisting of two UV lenses with focal 
lengths of $0.2 \mathrm{~m}$ and $1 \mathrm{~m}$ respectively. This telescope magnifies the beam by five times as indicated in Fig. 2b. A $50 \mu \mathrm{m}$ spatial filter was inserted at the focal spot between the two lenses to improve the homogeneity of the transverse profile. This causes an energy loss of $\sim 50 \%$.

The image at the position of the " target" was relayed to the photocathode, which is located $20 \mathrm{~m}$ away from the laser room.

\section{System performance}

After the upgrade was completed, the performance of the entire laser system was checked, including shot to shot fluctuations, the transverse and longitudinal profile of the UV beam, the operation of the pulse stacker and of the transport line to the cathode.

\subsection{Shot to shot fluctuations}

An important aspect of the laser performance is its repeatability from shot to shot (on the $1 \mathrm{~Hz}$ scale) as this determines the charge fluctuations in the electron beam. The single seed pulse after the pulse picker was scrutinized with a fast photodiode. At $1 \mathrm{~Hz}$ repetition rate, the amplitude of more than 50,000 pulses was recorded and the ratio between the standard deviation and the mean was measured to be $1.3 \%$; this is the irreducible noise level, which originates in the seed laser. The pulse fluctuations after the multi-pass amplifier were measured by averaging more than 100 shots and dividing the standard deviation by the mean. The results were recorded for a different number of round trips and are shown in Fig. 7. The open circles are the measured data and the noise for the "zero" round trip was set at $1.3 \%$ as determined from the seed laser. To identify 
the source of the noise, we decouple the fluctuations originating in the seed laser from the fluctuations introduced by the multi-pass amplifier. Assuming that these fluctuations are independent and therefore add quadratically, we write

$$
\text { Fluc }_{\text {MP }}=\sqrt{\text { Fluc }_{\text {Measured }}^{2}-\text { Fluc }_{\text {Laser }}^{2}} \text {. }
$$

Here, Fluc ${ }_{\text {Measured }}$ are the fluctuations measured with the photodiode and Fluc $_{\text {Laser }}$ is the

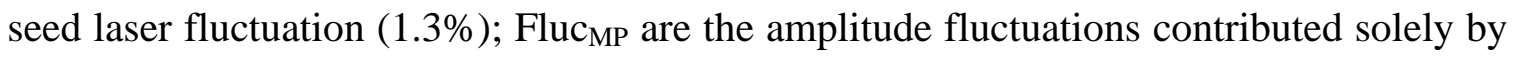
the multi-pass amplifier.

The resulting fluctuations in the multi-pass are shown by the squares in Fig. 7. They were fit by a straight line with a slope of $0.64 \%$, which implies that each round trip adds $0.64 \%$ to the amplitude fluctuation in the final output. This level for the fluctuation added in a single round trip is consistent with the voltage stability of the power supply that drives the multi-pass amplifier flash lamps. According to our measurement, the voltage fluctuations of the power supply are less than $0.2 \%$ from shot to shot; namely fluctuations in power of $<0.4 \%$, or $<0.8 \%$ for each round trip, in agreement with the data shown in Fig. 7.

After the multi-pass amplifier, the pulse was coupled to the 2-pass amplifiers and no significant increase of the fluctuations was observed. The reason is that the multi-pass amplifier and 2-pass amplifiers are independent of each other so their noise contributions add quadratically. Furthermore in each 2-pass amplifier (they use similar power supplies as the multi-pass amplifier), the pulse experiences only one round trip, which adds negligibly to the fluctuations of the final output.

The shot to shot fluctuations were monitored at all the stages along the beam path. They were measured after the multi-pass amplifier, after the 2-pass amplifiers, after the 
frequency doubling crystal, and in the UV. This is shown in Fig. 8, where the shot to shot fluctuations of the pulse amplitude after the multi-pass amplifier and after the UV crystal recorded for the entire month of July 2005 are plotted on the same graph. In all cases we used an integrating power meter to measure the power in the pulse train. The amplitude fluctuations of the UV output follow closely the fluctuations after the multipass amplifier. We conclude that the multi-pass amplifier is the dominant noise source in the entire laser system. Reduction of the shot to shot fluctuations beyond the present level could possibly be achieved by replacing the multi-pass amplifier with a diode pumped system.

\subsection{Transverse and longitudinal profiles}

The transverse uniformity of the laser beam on the photocathode is crucial for generating a high quality electron beam with low emittance [12]. The image of the UV spot was measured in the laser room and could also be observed on a "virtual cathode" at the location of the RF gun. Furthermore by inserting a test pattern in the beam in the laser room we could ascertain that the image was faithfully relayed to the virtual cathode.

After the frequency quadrupling crystal, the beam is directed by the mirrors and UV telescope to the location of the diagnostic "target" as shown in Fig. 2b. The telescope consists of two UV lenses with focal lengths of $0.2 \mathrm{~m}$ and $1 \mathrm{~m}$ respectively. To remove high spatial frequency components from the beam a $50 \mu \mathrm{m}$ diameter spatial filter was inserted at the focal spot between the two lenses. Figure 9 shows the UV image on the "target” taken with a CCD camera. The beam profile is close to a Gaussian and has 
FWHM $4.3 \mathrm{~mm}$, which is well suited for most applications. In case a smaller beam size is desired, an iris can be inserted at the location of the "target".

The longitudinal profile of a single UV pulse was measured with a streak camera that has time resolution of $\sim 2$ ps. No pulse length fluctuations were observed and a typical longitudinal profile of a single UV pulse obtained by the streak camera is shown in Fig. 10. The UV pulse length is determined to be $5.4 \mathrm{ps}$, practically identical to the IR seed pulse length. This can be understood by the fact that both the frequency doubling and frequency quadrupling crystals are operating in the deep saturation regions, and therefore no pulse narrowing should be expected from either conversion process.

\subsection{Pulse stacker and transport to the cathode}

Simulations show [13] that for an electron bunch with a flat top in the time domain, the space-charge induced emittance growth is reduced. Since a single UV pulse is Gaussian, we built a pulse stacker so that four UV pulses can be stacked together with the same amplitude and fixed time delay between them.

The schematic diagram of the pulse stacker [14] is shown in Fig. 11. In the setup, the linearly polarized input of the pulse stacker is rotated $45^{\circ}$ by a half-wave plate so that it contains both P and S components equally. Then the beam is split by the first (UV coated) cube polarizer, which passes the $\mathrm{P}$ component of the beam and reflects the $\mathrm{S}$ component. The two components follow different optical paths. One path has fixed optical length while the other one has an adjustable optical delay. The two separated beams are recombined by the second cube polarizer into a single beam, which consists of two pulses with the desired delay and S and P polarizations respectively. After passing through 
another similar set of half-wave plate, two cube polarizers, and adjustable differential optical paths, the beam eventually has four pulses stacked.

In the output beam of the pulse stacker, the four pulses are polarized in the sequence of S, P, S and P. The orthogonal polarizations eliminate any interference between the neighbors both transversely and longitudinally. Interference would have introduced unwanted patterns on the photocathode. A fixed optical delay of $\sim 5$ ps was introduced between adjacent pulses, and the stacked pulses were measured with the streak camera in the time domain. The result is shown in Fig. 12, including a fit to four combined identical Gaussians with 5 ps FWHM delayed by 5.2 ps with respect to each other. While the main features of the experimental result follow the fitted curve, the measured rise time of 6.4 ps (10\% to $90 \%)$ is slightly longer than expected. This may be caused by the fact that we are limited by the 2 ps time resolution of the streak camera, which will smooth out any sharp rise or fall times.

To relay the UV beam from the laser room onto the photocathode, which is located more than $20 \mathrm{~m}$ away, an imaging system was installed along the beam transport line. The beam transport line is folded and there are only three turning corners, where the optics can be mounted in the boxes. Figure 13 shows the schematic of the transport line (as a 2-dimensional projection). A UV coated standard singlet spherical plano-covex lens with focal length of $5 \mathrm{~m}$ was inserted in front of the flat mirror in turning box 2. Finally, we can introduce masks in the laser room, so that the transverse pattern on the photocathode has any desired configuration. This feature is used to investigate the dependence of the emittance on non-uniform electron emission in the transverse plane, and to benchmark the simulation code of the electron beam line [15]. 


\subsection{Long pulse train}

As mentioned before, the laser system is able to deliver up to 800 pulses. A long train consisting of 400 seed pulses after the pulse picker is shown in Fig. 14a. Because of the stable output of the seed laser, the train envelope is extremely flat. However, after amplification in the multi-pass and the two 2-pass amplifiers, the shape of the pulse train is distorted as shown in Fig. 14b.

This is due to variation of the overall gain, as the stored energy in the amplifying media is depleted. To achieve a flat envelope over the entire pulse train, it is possible to pre-shape the seed pulse train with a slow Pockels cell, so as to anticipate the subsequent gain variations [6].

In addition to the envelope distortion, the train in Fig. 14b shows pulse to pulse fluctuations of order $10 \%$ and possibly with a period of $\mathrm{T} \sim 50 \mu \mathrm{s}$. These fluctuations may be due to thermal effects in the rods and are under investigation. 


\section{Acknowledgements}

We thank our colleagues in the A0 group for their assistance and contributions during the course of this work, in particular Drs. G. Blazey, H. Edwards, and Ph. Piot for supporting this upgrade. Dr. N. Barrov and Mr. J. Santucci participated in laser related activities. This work was supported in part by DOE contract DE-AC02-76CH00300 at Fermilab, by DOE grant DEFG0291ER40685 at the University of Rochester and by Northern Illinois University. 
[1] J. P. Carneiro et al., Beam transport, acceleration and compression studies in the Fermilab high-brightness photo-injector, Proceedings of the XIX International Linac Conference, C. E. Eyberger, R. C. Pardo, \& M. M. White, Editors, ANL-98/28, Argonne National Laboratory, Argonne, Illinois, 1998, p. 878-880.

[2] The laser specifications can be found on the website of the company. http://www.tbwp.com/Time_Bandwidth/Sites/S_Products/FrameSetPico/GE 100.htm.

[3] Conoptics, Inc., m360-80 modulator and model 25D HV driver.

[4] I. Will et al, IEEE Journal of Quantum Electronics, Vol 34, NO. 10 (1998) 2020-2028.

[5] I. Will et al, Nuclear Instruments and Methods A, 541 (2005) 467-477.

[6] A. R. Fry, M. J. Fitch, A. C. Melissinos, B. D. Taylor, Nucl. Instr. And Meth. A 430 (1999) 180.

[7] P. Laporta, V. Magni, and L. Pallaro, Optics Communications, 51 (1984) 95.

[8] M. J. Fitch, "Electro-Optic Sampling of Transient Electric Fields from Charged Particle Beams”, Ph. D. dissertation, University of Rochester, 2000.

[9] Hewlett-Packard HP70951A Optical Spectrum Analyzer on loan from R. Pasquinelli (FNAL).

[10] Conoptics, Inc., m360-105 modulator and model 25D HV driver.

[11] A. R. Fry, "Novel Pulse Train Glass Laser for RF Photoinjectors”, Ph. D. dissertation, University of Rochester, 1996. 
[12] F. Zhou, I. Ben-Zvi, M. Babzien, X. Y. Chang, A. Doyuran, R. Malone, X. J. Wang and V. Yakimenko, Phys. Rev. Special Topics, Vol. 5, 094203-1 (2002).

[13] Ph. R. Piot et al, "Upgrade of the Fermilab/NICADD Photoinjector Laboratory", PAC05 paper TPPE044.

[14] S. Schreiber, TESLA Collaboration Meeting, WG3, 2004

[15] Jianliang Li, Philippe Regis-Guy Piot, Rodion Tikhoplav, "Production of Transverse Controllable Laser Density Distribution in Fermilab/NICADD Photoinjector", PAC05 Paper WPAP043. 


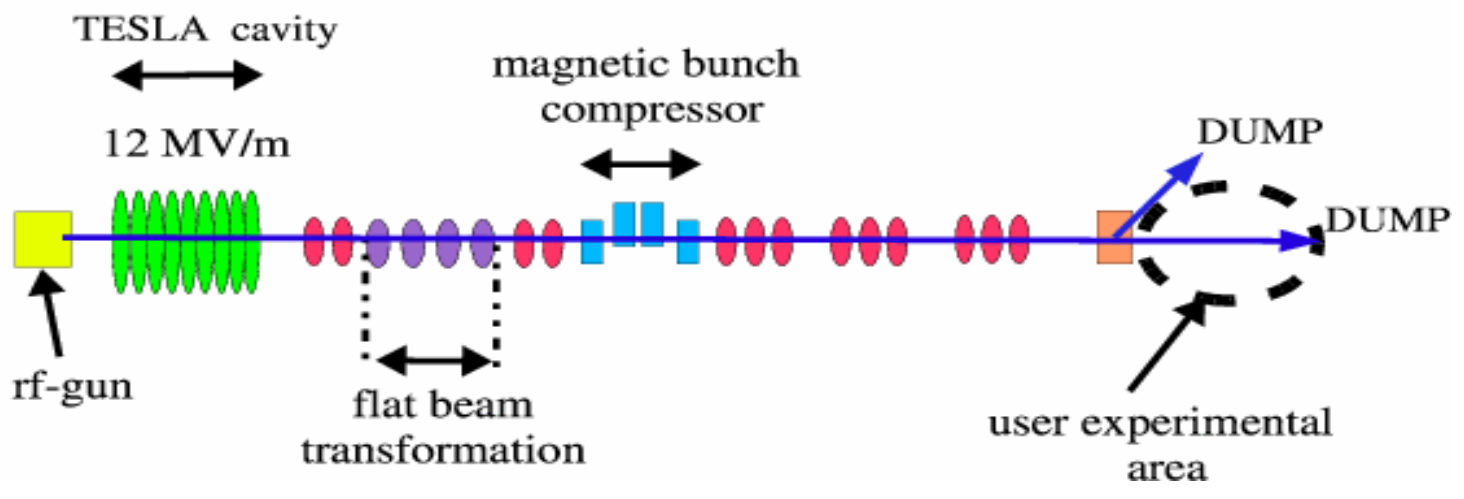

Fig. 1 


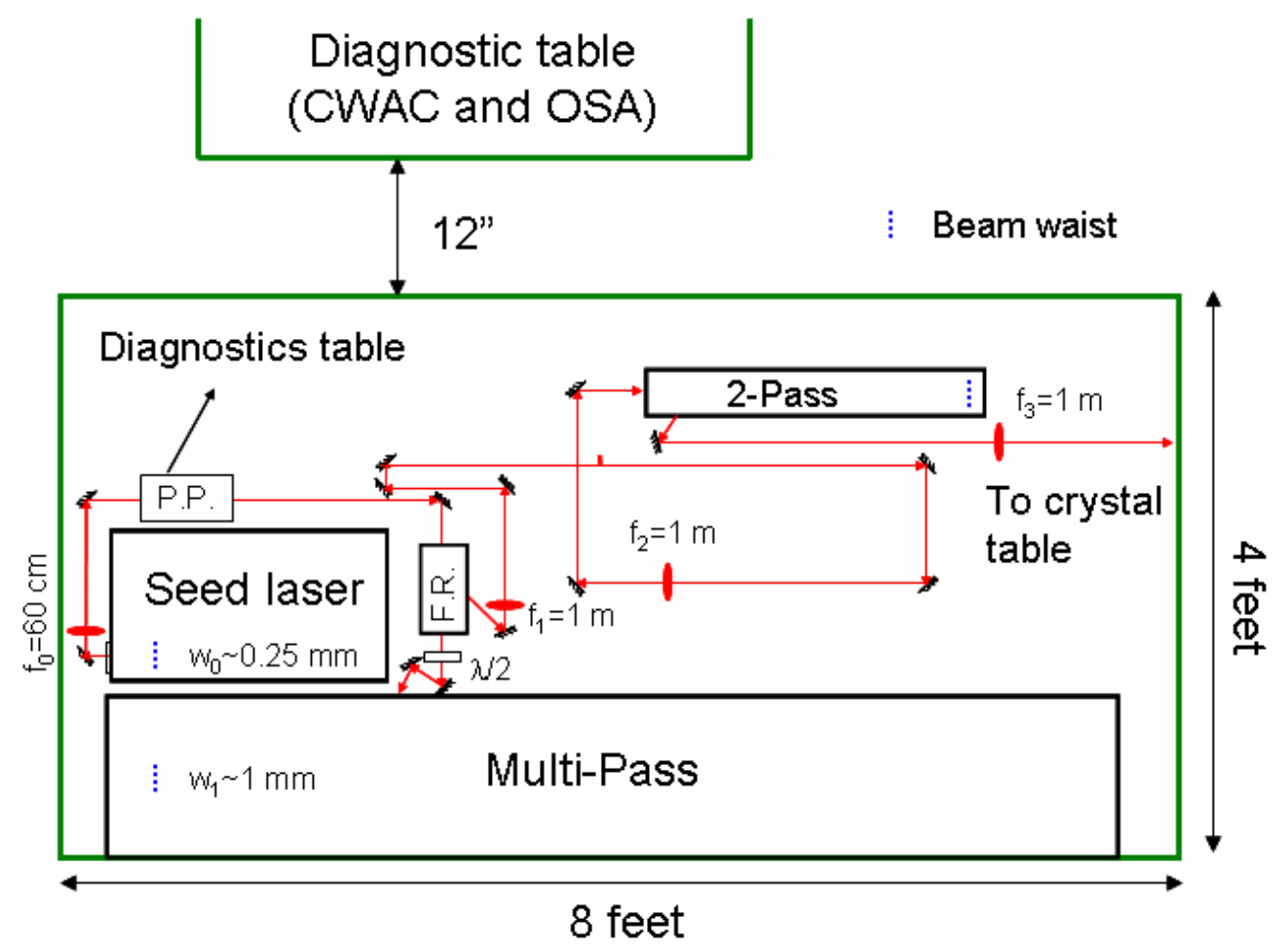

(a)

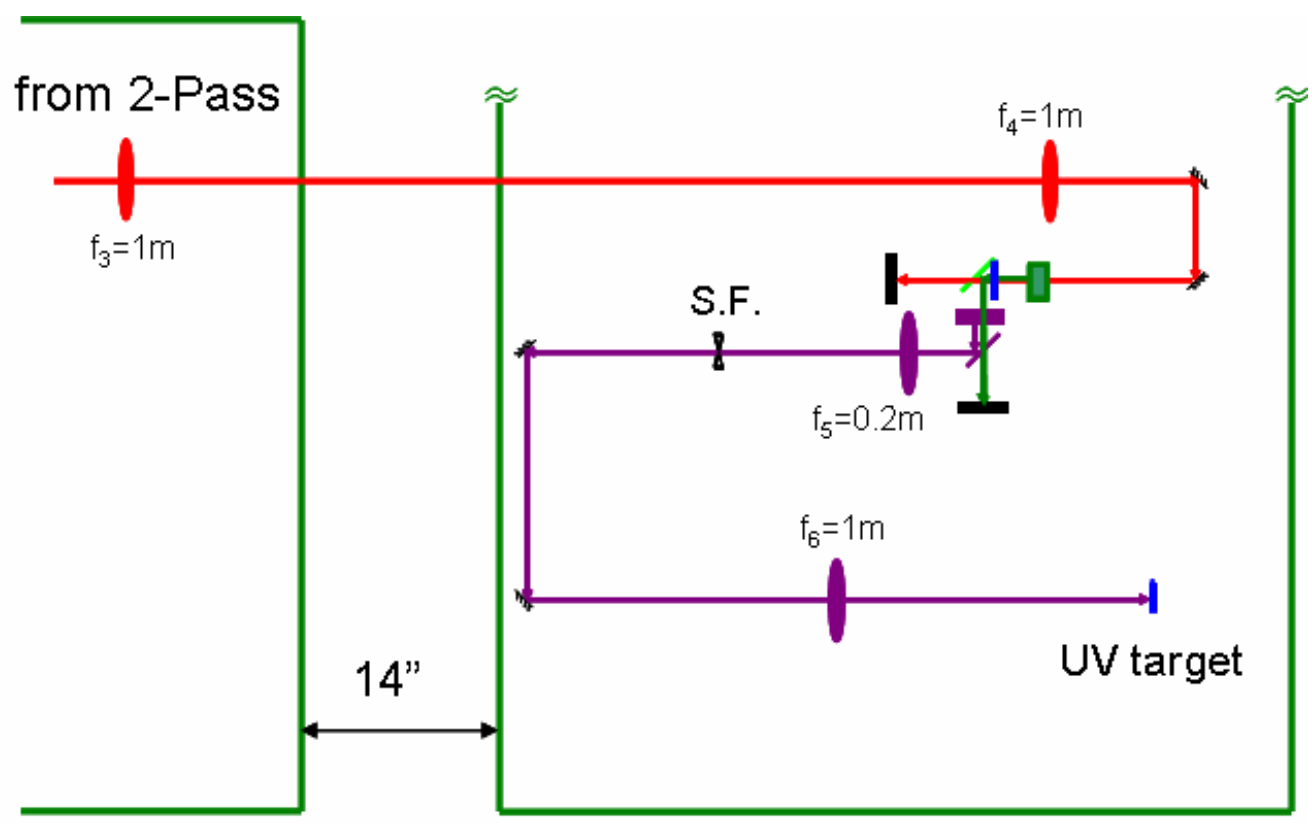

(b)

Fig. 2 (a) and (b) 


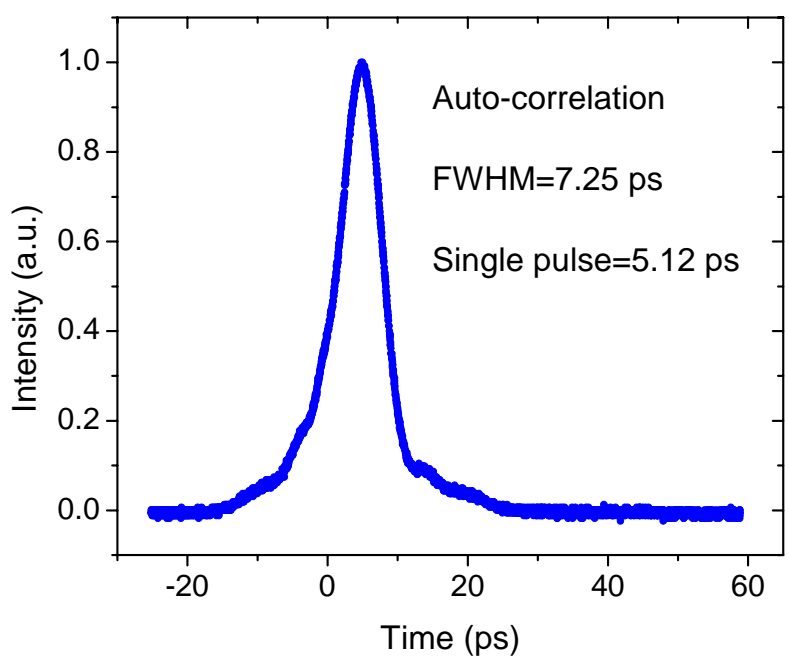

(a)

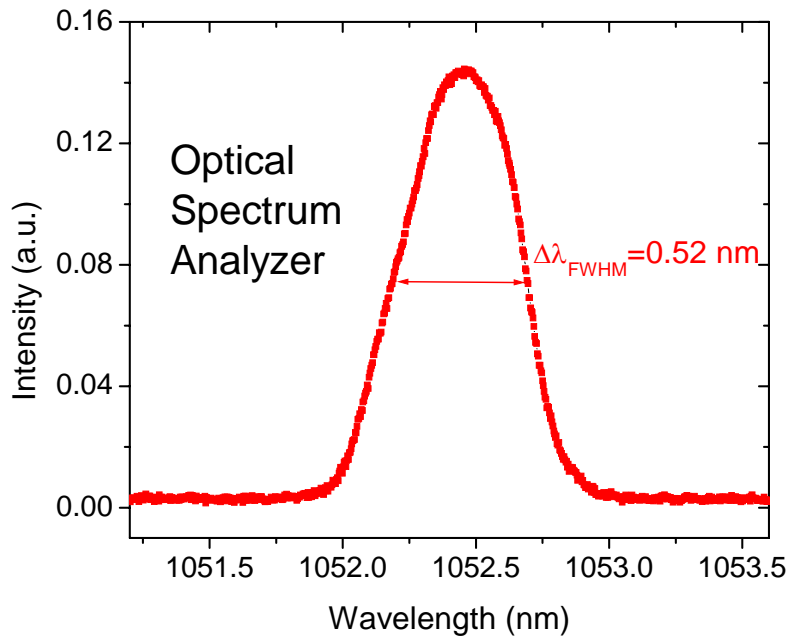

(b)

Fig. 3 (a) and (b) 


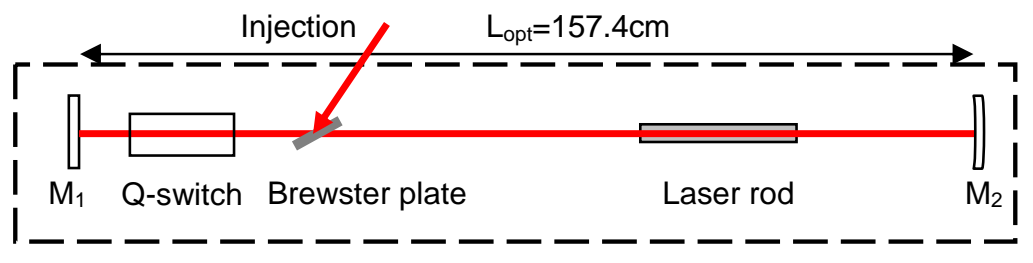

(a)

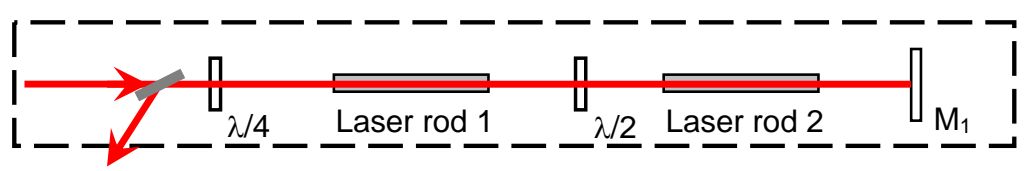

(b)

Fig. 4 (a) and (b) 


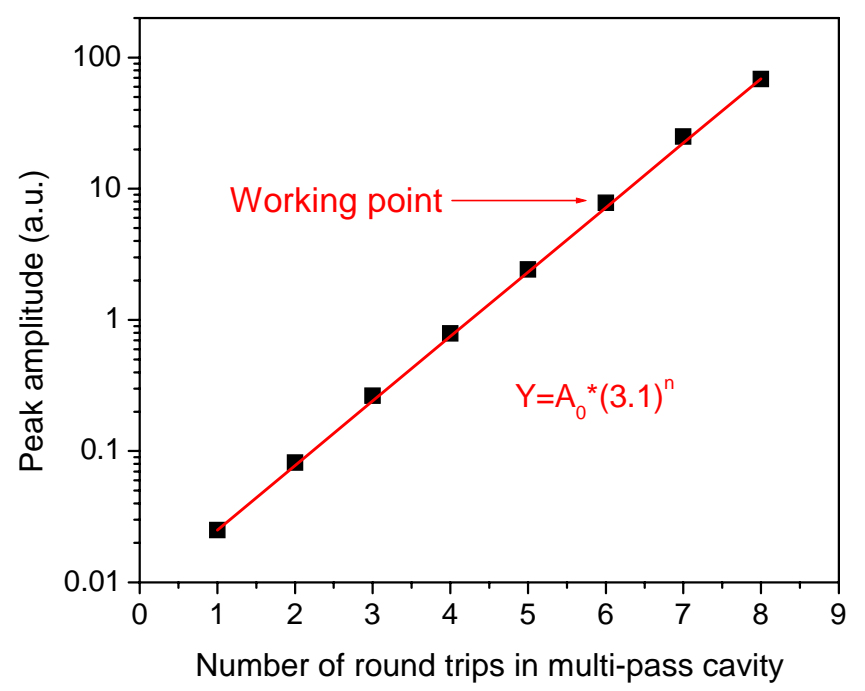

Fig. 5 


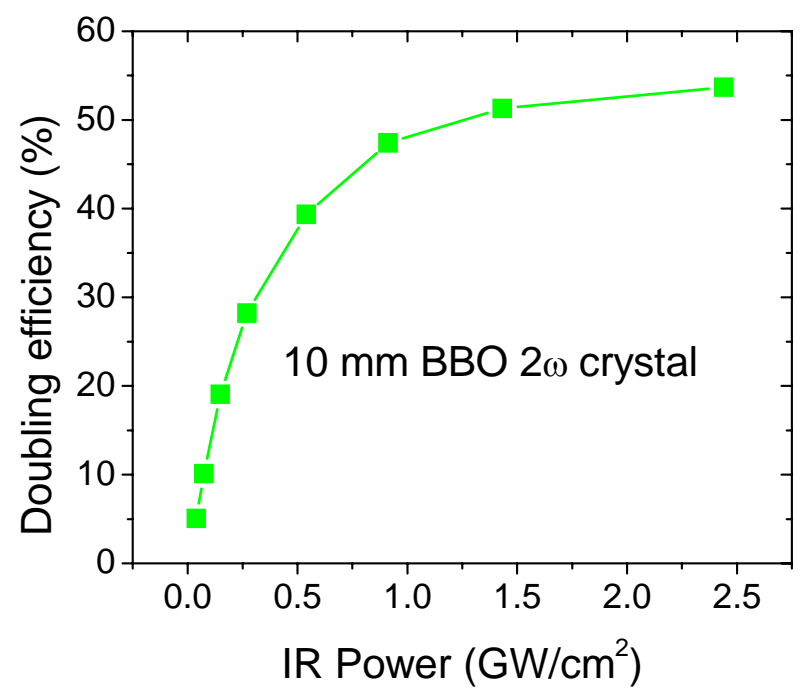

(a)

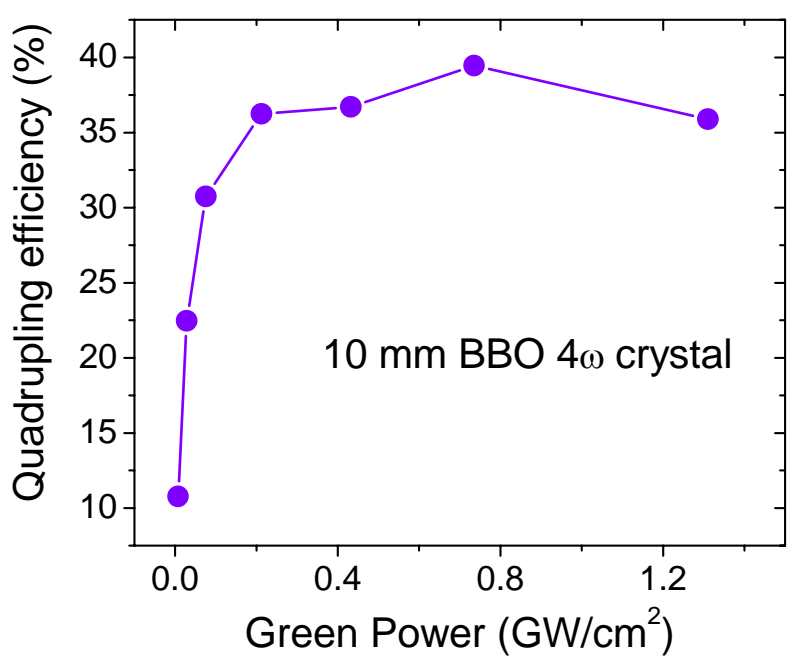

(b)

Fig. 6 (a) and (b) 


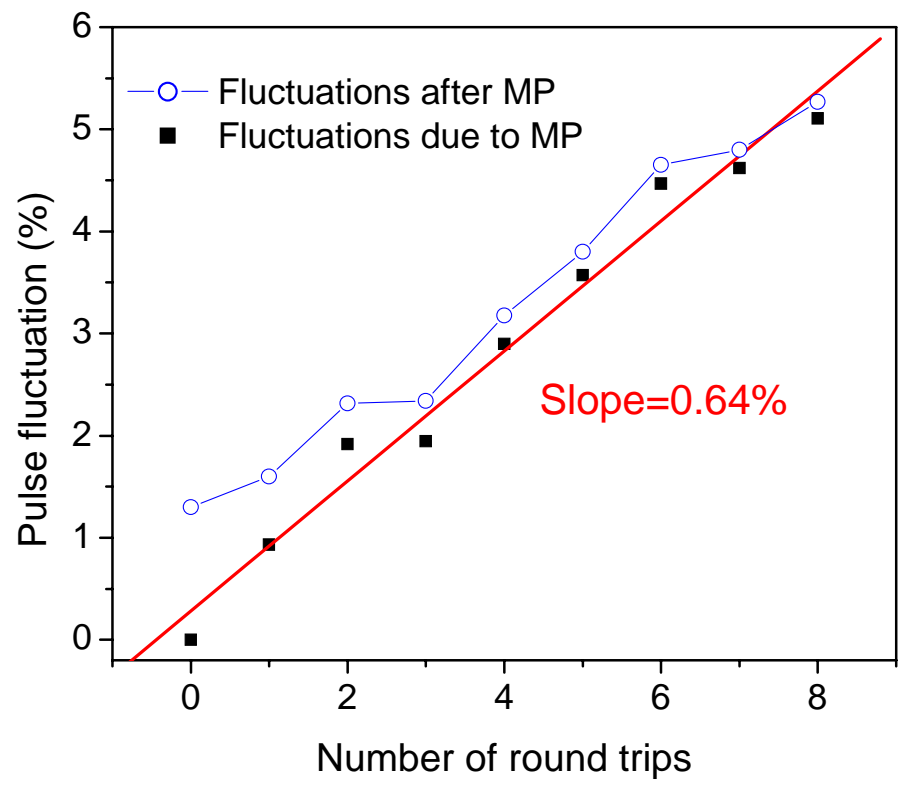

Fig. 7 


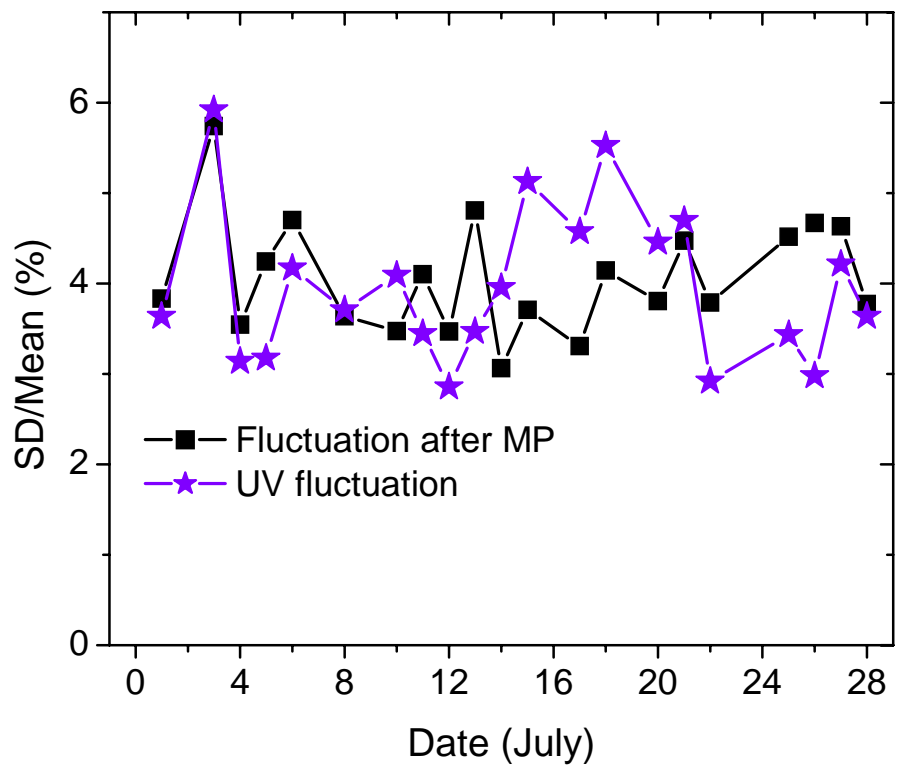

Fig. 8 

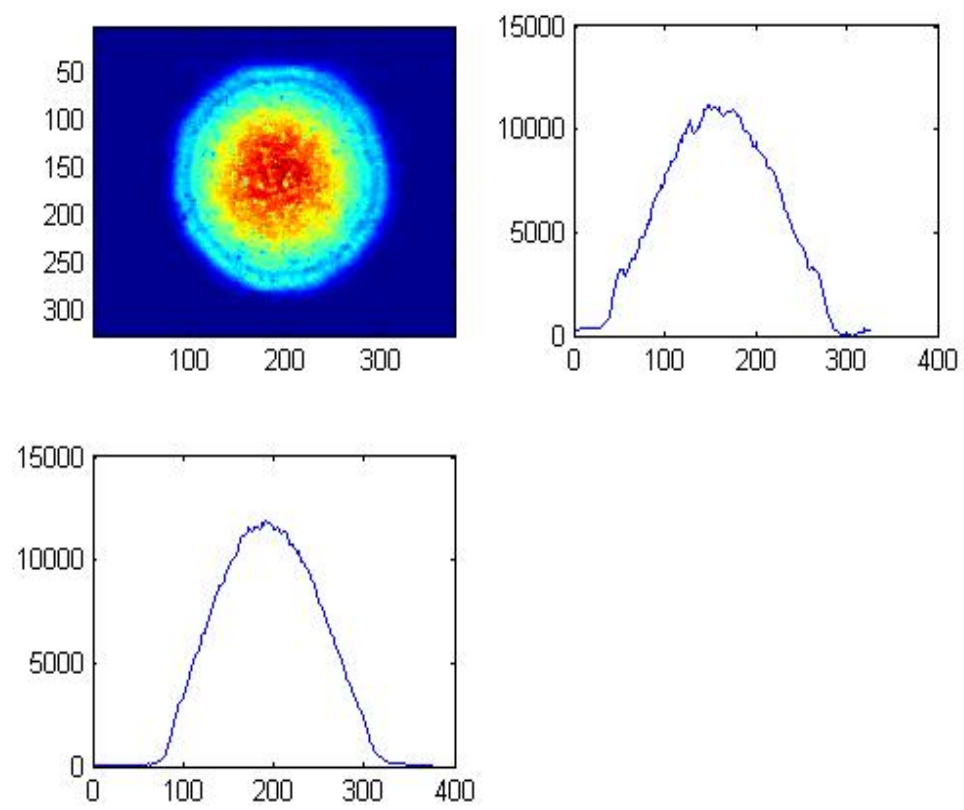

Fig. 9 


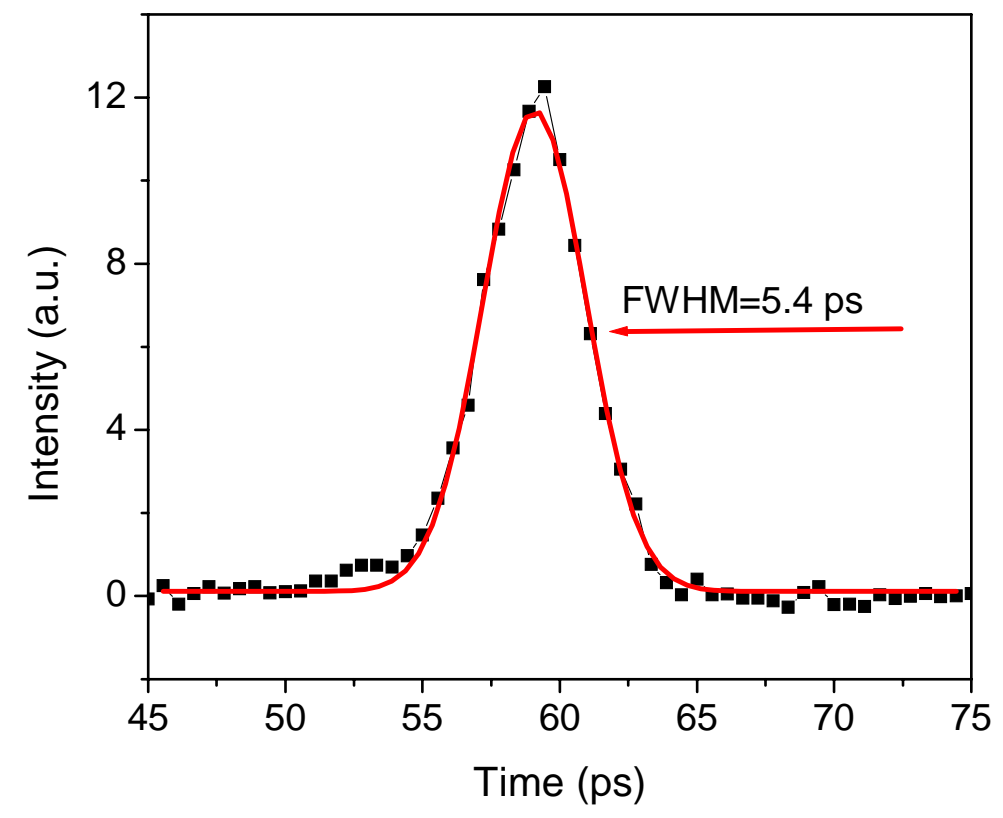

Fig. 10 


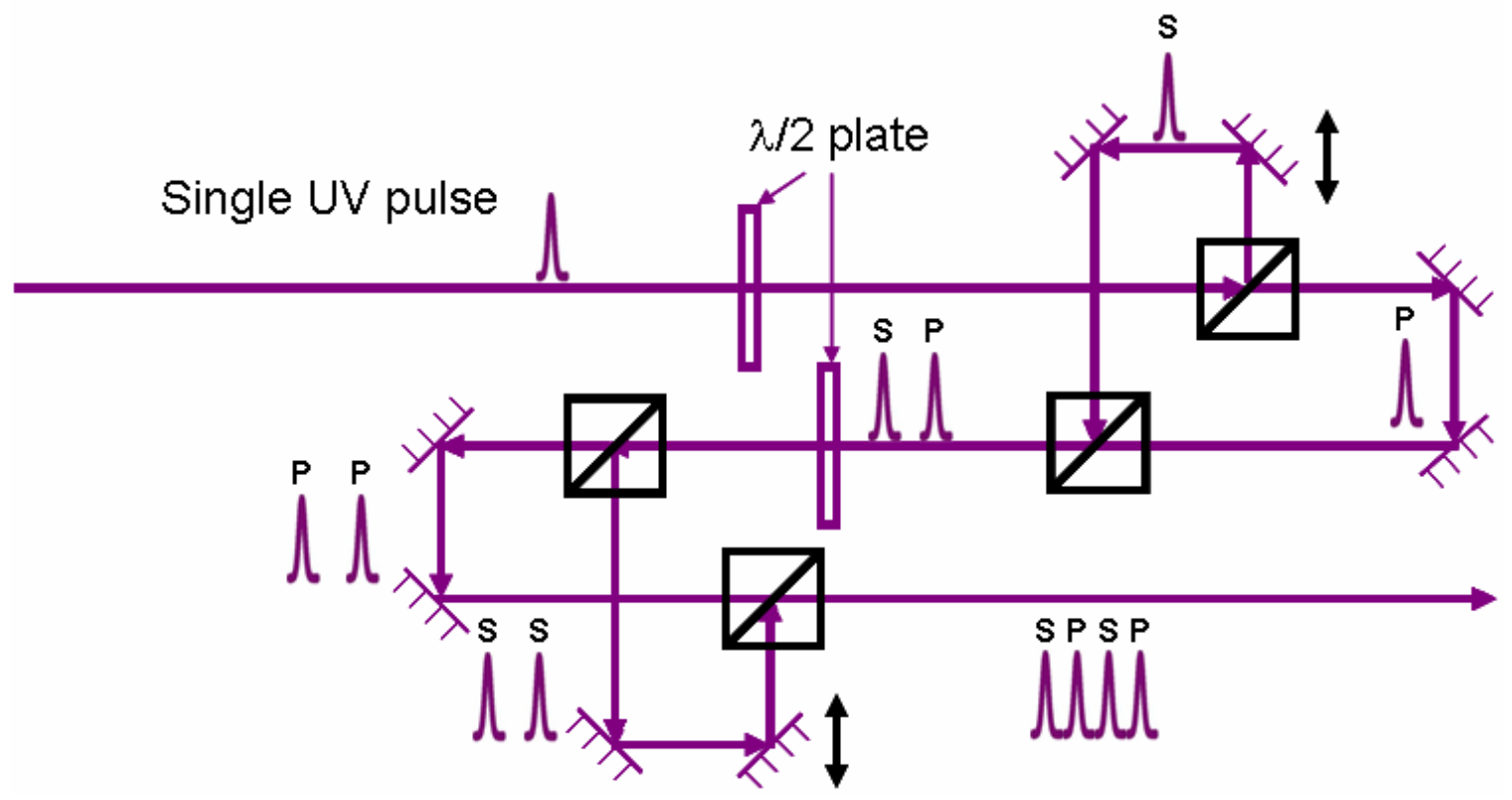

Fig. 11 


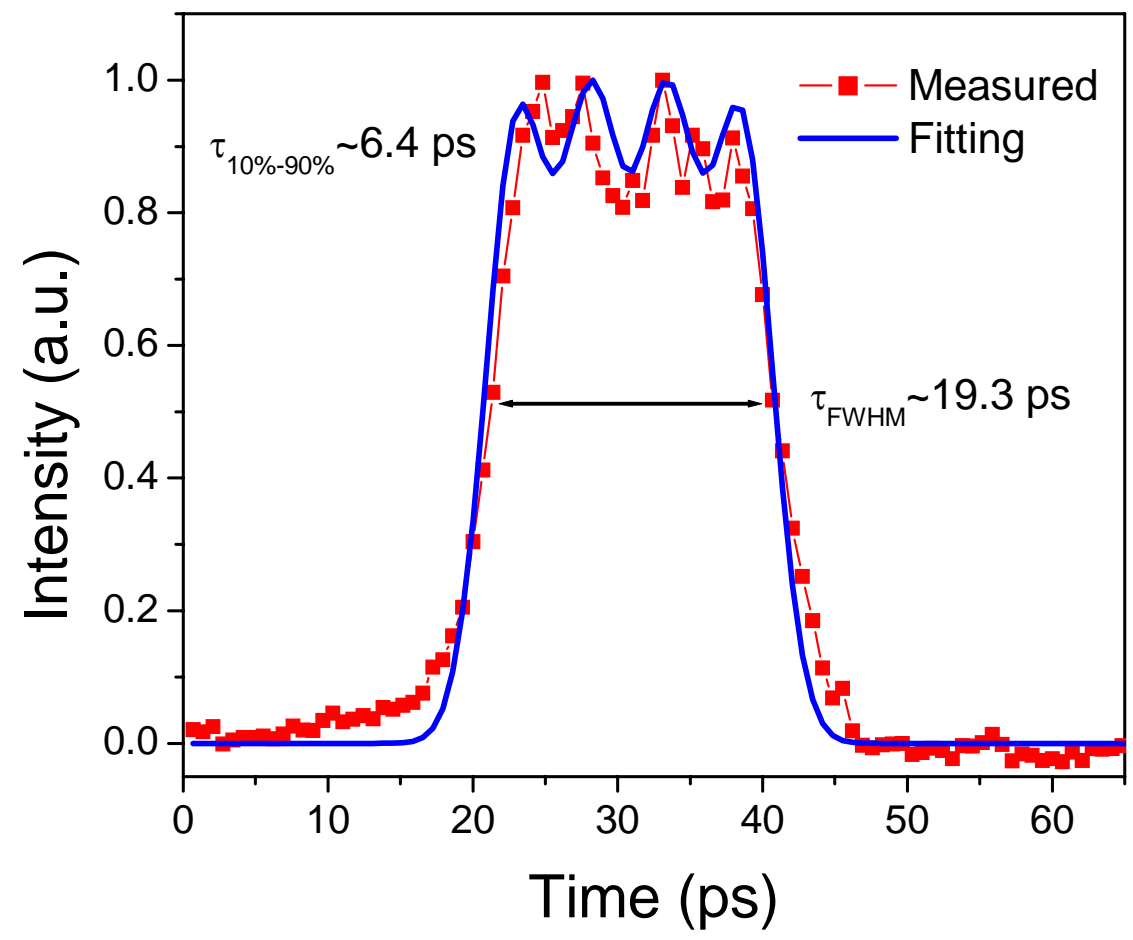

Fig. 12 


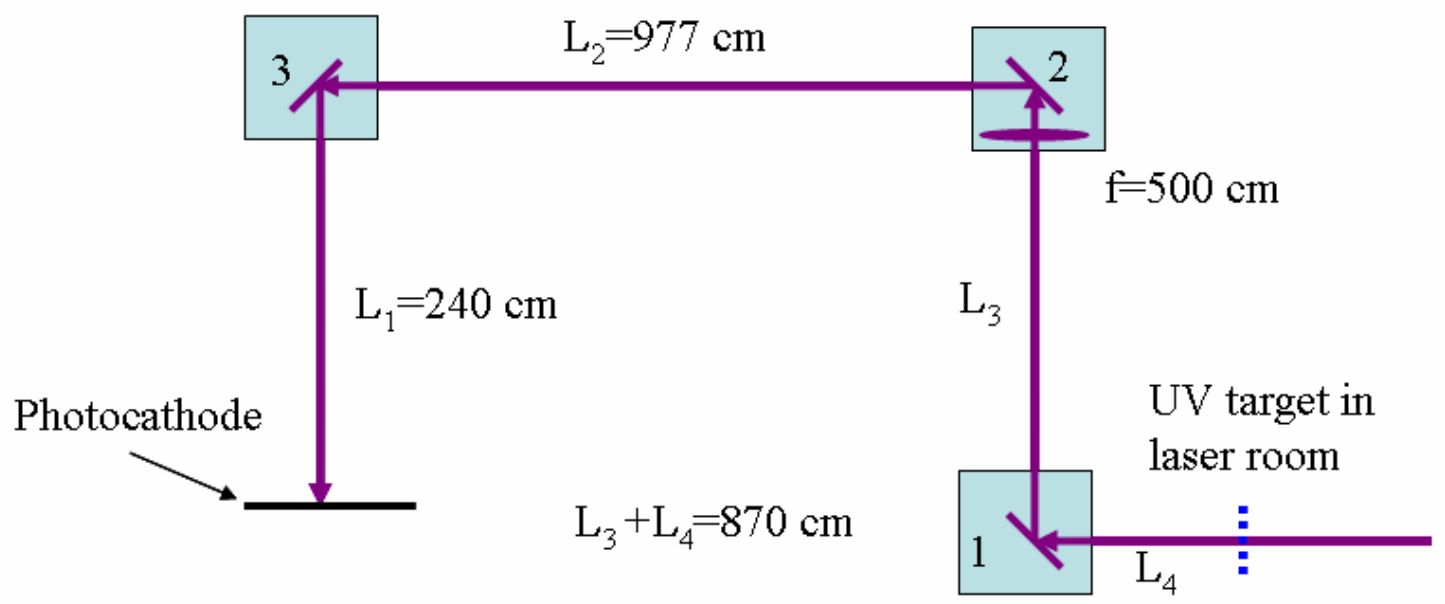

Fig. 13 


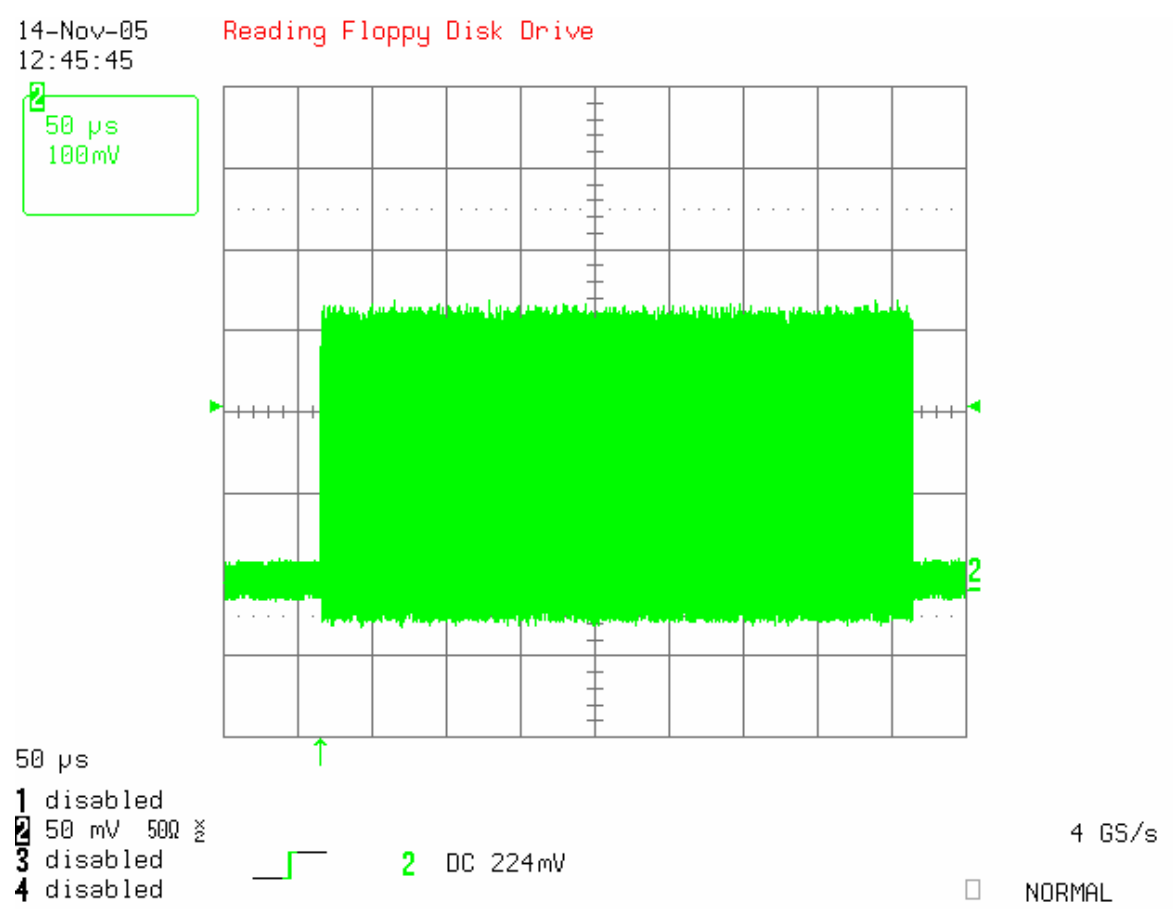

(a)

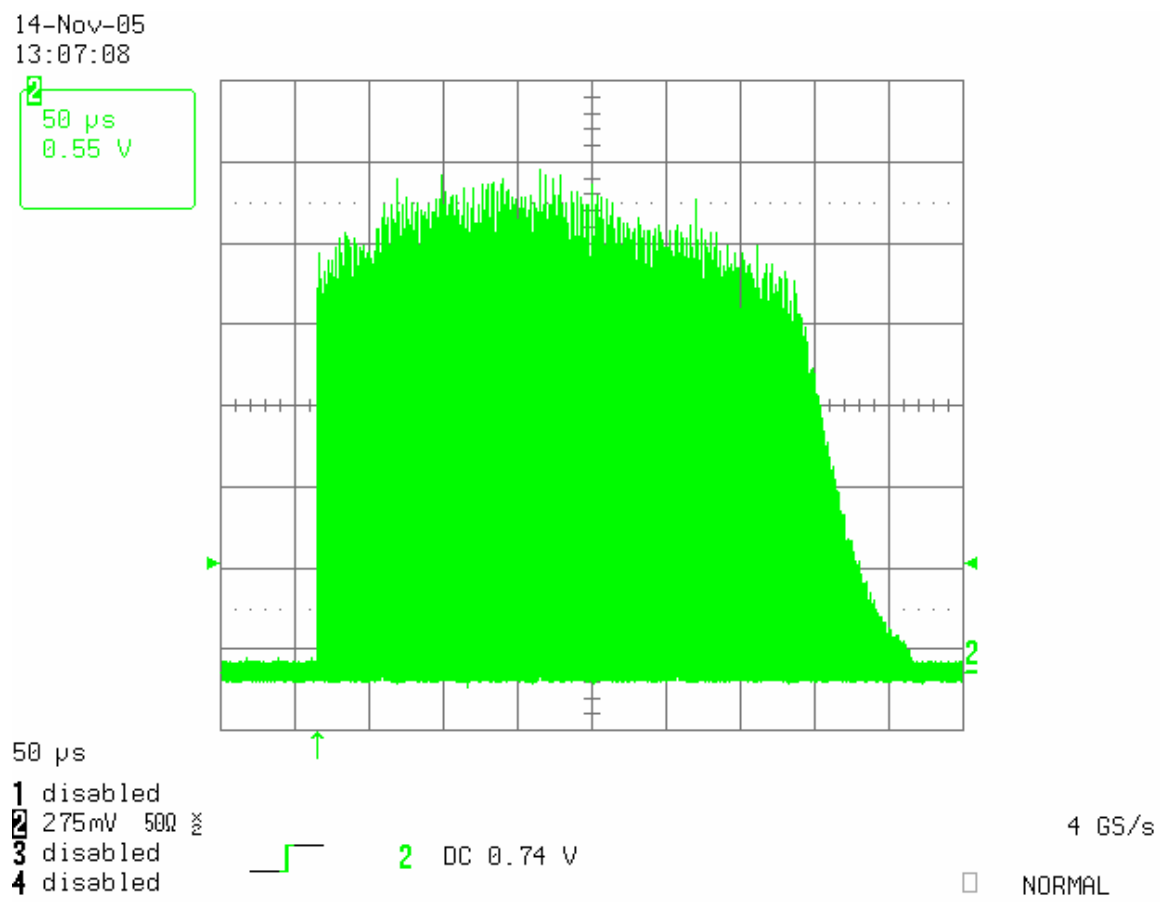

(b)

Fig. 14 
Table I. Laser parameters

\begin{tabular}{|l|l|}
\hline Oscillator frequency & $81.25 \mathrm{MHz}$ \\
\hline Oscillator wavelength & $1054 \mathrm{~nm}$ \\
\hline Oscillator energy/pulse & $5.5 \mathrm{~nJ}$ \\
\hline Energy/pulse after multi-pass & $6 \mu \mathrm{J}$ \\
\hline Energy/pulse after two-pass & $100 \mu \mathrm{J}$ \\
\hline UV energy/pulse after crystals & $20 \mu \mathrm{J}$ \\
\hline UV energy/pulse on cathode & $10 \mu \mathrm{J}$ \\
\hline UV pulse length (FWHM) & $5 \mathrm{ps}$ \\
\hline Separation of pulses in train & $1 \mu \mathrm{s}$ \\
\hline Length of pulse train & up to $800 \mathrm{pulses}$ \\
\hline Repetition rate & $1 \mathrm{~Hz}$ \\
\hline
\end{tabular}


Figure captions:

Fig. 1. Schematic overview of the photoinjector at A0.

Fig. 2. Layout of the laser system, amplifier table (a) and crystal table (b). PP designates a Pulse Picker; FR, Faraday Rotator; 2P, two-pass amplifier; SF, spatial filter.

Fig. 3. (a) Autocorrelation as measured with CWAC. The FWHM was determined to be 7.23 ps, which implies an IR pulse length of 5.12 ps assuming a Gaussian profile of the input pulse. (b) Spectrum of the seed laser as measured by the HP70951A Optical Spectrum Analyzer (OSA). The FWHM was determined to be $0.52 \mathrm{~nm}$.

Fig. 4. (a) Schematic top view of the multi-pass cavity. (b) Schematic top view of the 2pass amplifiers.

Fig. 5. Single pulse amplitude measured with a fast photodiode versus the number of round trips in the multi-pass cavity.

Fig. 6. Efficiency of the doubling crystal (a) and efficiency of the quadrupling efficiency (b) for different input pulse intensity.

Fig. 7. Pulse to pulse fluctuations after the multi-pass amplifier measured for a different number of round trips.

Fig. 8. Pulse to pulse fluctuations after the multi-pass amplifier and after the UV crystal for the entire month of July 2005.

Fig. 9. UV beam profile measured on the target in the laser room. A $50 \mu \mathrm{m}$ spatial filter was inserted to improve the UV transverse profile. The beam size is about $4.3 \mathrm{~mm}$ of FWHM.

Fig. 10. Picture of a single UV pulse recorded by the streak camera (left). Projection of the image on the vertical axis (right). 
Fig. 11. Schematic diagram of a UV pulse stacker with four orthogonally polarized pulses.

Fig. 12. Four stacked UV pulses. The squares are the data as measured by a streak camera and the solid line is a fit to four Gaussians of equal amplitude and of 5 ps width, combined with a delay of 5.2 ps between neighbors.

Fig. 13. Schematic diagram of the UV beam transport line.

Fig. 14. (a) A long pulse train of 400 seed pulses captured with a fast digital oscilloscope;

(b) The same long pulse train after the two-pass amplifiers. 